\title{
Postcranioplasty seizures following decompressive craniectomy and seizure prophylaxis: a retrospective analysis at a single institution
}

\author{
Mun-Chun Yeap, MD, Ching-Chang Chen, MD, Zhuo-Hao Liu, MD, PhD, Po-Chuan Hsieh, MD, \\ Cheng-Chi Lee, MD, PhD, Yu-Tse Liu, MD, Alvin Yi-Chou Wang, MD, Yin-Cheng Huang, MD, PhD, \\ Kuo-Chen Wei, MD, Chieh-Tsai Wu, MD, and Po-Hsun Tu, MD
}

\author{
Department of Neurosurgery, Chang Gung Memorial Hospital, Linkou Medical Center, Chang Gung Medical College \& \\ University, Taoyuan City, Taiwan
}

\begin{abstract}
OBJECTIVE Cranioplasty is a relatively simple and less invasive intervention, but it is associated with a high incidence of postoperative seizures. The incidence of, and the risk factors for, such seizures and the effect of prophylactic antiepileptic drugs (AEDs) have not been well studied. The authors' aim was to evaluate the risk factors that predispose patients to postcranioplasty seizures and to examine the role of seizure prophylaxis in cranioplasty.

METHODS The records of patients who had undergone cranioplasty at the authors' medical center between 2009 and 2014 with at last 2 years of follow-up were retrospectively reviewed. Demographic and clinical characteristics, the occurrence of postoperative seizures, and postoperative complications were analyzed.

RESULTS Among the 583 patients eligible for inclusion in the study, 247 had preexisting seizures or used AEDs before the cranioplasty and 336 had no seizures prior to cranioplasty. Of these 336 patients, $89(26.5 \%)$ had new-onset seizures following cranioplasty. Prophylactic AEDs were administered to 56 patients for 1 week after cranioplasty. No early seizures occurred in these patients, and this finding was statistically significant $(p=0.012)$. Liver cirrhosis, intraoperative blood loss, and shunt-dependent hydrocephalus were risk factors for postcranioplasty seizures in the multivariable analysis.
\end{abstract}

CONCLUSIONS Cranioplasty is associated with a high incidence of postoperative seizures. The prophylactic use of AEDs can reduce the occurrence of early seizures.

https://thejns.org/doi/abs/10.3171/2018.4.JNS172519

KEYWORDS cranioplasty; craniectomy; seizure; seizure prophylaxis; complication; surgical technique

$\mathrm{C}$ RANIOPLASTY, the placement of a bone flap or prosthesis to repair a defect in the skull, is a surgical procedure usually performed in patients who have undergone decompressive craniectomy (DC) due to poor control of elevated intracranial pressure following traumatic brain injury (TBI) or stroke. Though it is a relatively simple procedure, many complications, such as hemorrhage, infection, and bone necrosis, have been reported. ${ }^{11,14,22,27}$ In addition, seizures are common after cranioplasty and are associated with poor outcomes; however, the risk factors for, and the incidence of, such seizures have not been well described. Moreover, there are few reports about the effect of antiepileptic drugs (AEDs) for seizure prophylaxis. ${ }^{15}$ Our aim was to evaluate the risk factors that predispose patients to postcranioplasty seizures and to examine the role of seizure prophylaxis in cranioplasty.

\section{Methods \\ Study Design}

The records of patients who had undergone cranioplasty at a single medical center during the period from January 2009 to December 2014 and who had at least 2 years of follow-up were retrospectively reviewed. The study included all patients who had initially undergone DC for brain swelling due to TBI, hemorrhagic or ischemic stroke, or brain tumor or abscess. To standardize the baseline risks and pathological entities, the study excluded patients

ABBREVIATIONS AED = antiepileptic drug; $D C=$ decompressive craniectomy; $P M M A=$ polymethylmethacrylate; $T B I=$ traumatic brain injury . SUBMITTED October 6, 2017. ACCEPTED April 12, 2018.

INCLUDE WHEN CITING Published online September 21, 2018; DOI: 10.3171/2018.4.JNS172519.

${ }^{*}$ M.C.Y. and C.C.C. contributed equally to this study and share first authorship. 
who had undergone craniectomy for bone flap infections following craniotomy for all causes; neoplastic etiologies that involved the skull, such as meningioma and skull tumors; and cranioplasty for traumatic compound fractures. The institutional review board of Chang Gung Memorial Hospital approved the study.

\section{Study Variables}

Patient demographic data including age and sex were collected, as were data on the following medical comorbidities: hypertension, diabetes mellitus, end-stage renal disease, liver cirrhosis, and hydrocephalus before cranioplasty (permanent CSF shunt prior to and at the time of cranioplasty). Data on all operations and the duration of follow-up were recorded. Details of the initial DC were reviewed to identify the initial pathology. Extensive DC of at least $12 \times 14 \mathrm{~cm}$ along with dural augmentation was performed by experienced surgeons. A synthetic, suturable, nonabsorbable dural substitute (Neuro-Patch, Aesculap) was used as an artificial dural barrier. The type of material used to reconstruct the cranial defect was classified as autologous bone, 3D polymethylmethacrylate (PMMA; constructed with 3D printing technology), or titanium mesh (Biomesh, Bioplate Inc.; constructed with 3D printing technology). Blood loss during cranioplasty, repeat craniotomy before cranioplasty, and repeat cranioplasty were also recorded and analyzed. Complications related to cranioplasty were identified as postoperative intracranial hematoma (including subdural, epidural, and intraparenchymal hematoma) requiring reoperation, infection needing surgical debridement at the surgical site or abscess and empyema requiring reoperation, and hydrocephalus that developed and required a permanent CSF shunt after cranioplasty. All surgical procedures were performed by 10 neurosurgeons, each with over 5 years' experience performing craniectomy and cranioplasty. Two of the surgeons administered prophylactic AEDs for 1 week after cranioplasty.

A postoperative seizure was defined as any seizure that occurred after cranioplasty (early: $\leq 1$ week after operation, late: $>1$ week after operation). ${ }^{3}$ All patients who had undergone cranioplasty were divided into two groups: those with preexisting seizures before cranioplasty, and those with no seizures before cranioplasty. Seizure prophylaxis was defined as the prophylactic use of an AED after cranioplasty in patients who had no seizures before cranioplasty, and the drug was discontinued 1 week postoperatively if no seizures developed. Phenytoin or sodium valproate is the primary AED used at our center, and levetiracetam is only used when liver function impairment is identified prior to surgery. The dosage of AED for seizure prophylaxis is similar to that used in cases of traumatic head injury. Phenytoin was administered at a dose of $15-20 \mathrm{mg} / \mathrm{kg}$ by slow intravenous infusion before closure of the cranioplasty wound, followed by a 100-mg maintenance dose 3-4 times a day for 3 days, and then changed to $300 \mathrm{mg}$ orally once a day for the following 4 days. Sodium valproate was administered at a dose of $10-15 \mathrm{mg} / \mathrm{kg}$ by slow intravenous infusion before wound closure, then $10-60 \mathrm{mg} / \mathrm{kg}$ daily as a maintenance dose for 3 days, and then an oral form for the following 4 days. Levetiracetam was administered at a dose of $500 \mathrm{mg}$ by slow intravenous infusion before wound closure, and then a dose of $500 \mathrm{mg}$ twice daily.

\section{Statistical Analysis}

Baseline characteristics of the patients with and without seizures were compared using Fisher's exact test for categorical variables or a t-test for continuous variables (univariable analyses). The variables with a value of $p<0.2$ in the univariable analyses were input into a multivariable logistic regression model with backward selection. Finally, using Fisher's exact test, we compared the proportion of seizures in patients with and without AED use. A value of $p<0.05$ was considered statistically significant. Data analyses were conducted using SPSS version 22 (IBM Corp.).

\section{Results \\ Baseline Characteristics}

A total of 583 patients were included in this analysis. Of these patients, 247 had preexisting seizures or used AEDs before the cranioplasty and 336 patients had no seizures prior to the cranioplasty. Demographic and clinical data on the 336 patients without preexisting seizures before the cranioplasty are summarized in Table 1 . Of these 336 patients, 220 (65.5\%) had traumatic head injuries; 47 (14.0\%) had cerebral infarctions; 32 (9.5\%) had spontaneous intracerebral hemorrhage due to hypertension, arteriovascular malformations, aneurysms, or other vascular conditions; $28(8.3 \%)$ had brain tumors; and $9(2.7 \%)$ had spontaneous intracerebral infections (empyema or abscess). A total of $222(66.1 \%)$ patients received autologous bone flap reinsertion, $72(21.4 \%)$ received 3D PMMA, and 42 (12.5\%) received a titanium implant. Eighty-two patients had permanent hydrocephalus before cranioplasty. After cranioplasty, $20(6.0 \%)$ patients had postoperative infection and needed a repeat craniotomy, $5(1.5 \%)$ had postoperative acute hemorrhage, and 31 (9.2\%) had delayed hydrocephalus.

\section{Postcranioplasty Seizures}

Eighty-nine (26.5\%) patients experienced new-onset seizures. Univariable analyses showed that the initial reason for the DC, intraoperative blood loss, postoperative hemorrhage, and hydrocephalus before or after cranioplasty were associated with postcranioplasty seizures. Table 2 demonstrates the results of the multivariable analysis for new-onset seizures. Cirrhosis, intraoperative blood loss, and hydrocephalus were found to be risk factors for postcranioplasty seizures. Of the 336 patients without preexisting seizures, prophylactic AEDs were administered to $56(16.7 \%)$ for 1 week after cranioplasty. No early seizures occurred in these 56 patients. Phenytoin, sodium valproate, and levetiracetam were used in 37,16 , and 3 of the patients, respectively. The effect of prophylactic AEDs for new-onset postcranioplasty seizures is summarized in Table 3. Antiepileptic drug use showed statistical significance $(\mathrm{p}=0.012)$ toward protection against early seizures after cranioplasty. According to our results, the number needed to treat (NTT) is 8.9. That means that 8.9 seizures (early seizure) on average can be prevented because of the administration of AEDs. All drugs used were effec- 
TABLE 1. Characteristics of 336 patients without seizures before cranioplasty

\begin{tabular}{|c|c|c|c|}
\hline Variable & $\begin{array}{c}\text { New-Onset } \\
\text { Szs }\end{array}$ & No Szs & $\begin{array}{c}p \\
\text { Value }\end{array}$ \\
\hline No. of patients & $89(26.5 \%)$ & $247(73.5 \%)$ & \\
\hline Age in yrs & $44.7 \pm 17.5$ & $44.6 \pm 18.4$ & 0.977 \\
\hline Sex & & & 1.000 \\
\hline $\mathrm{F}$ & $29(32.6 \%)$ & $83(33.6 \%)$ & \\
\hline M & $60(67.4 \%)$ & $164(66.4 \%)$ & \\
\hline Initial reason for $\mathrm{DC}$ & & & 0.019 \\
\hline $\mathrm{ICH} \&$ vascular lesions & $11(12.4 \%)$ & $21(8.5 \%)$ & \\
\hline Infarction & $9(10.1 \%)$ & $38(15.4 \%)$ & \\
\hline Infection & $3(3.4 \%)$ & $6(2.4 \%)$ & \\
\hline Trauma & $63(70.8 \%)$ & $157(63.6 \%)$ & \\
\hline Tumor & $3(3.4 \%)$ & $25(10.1 \%)^{*}$ & \\
\hline $\begin{array}{l}\text { Interval btwn } 2 \text { proce- } \\
\text { dures in days }\end{array}$ & $245.5 \pm 491.9$ & $335.6 \pm 784.7$ & 0.374 \\
\hline Bone material & & & 0.065 \\
\hline 3D PMMA & $12(13.5 \%)$ & $60(24.3 \%)$ & \\
\hline Autologous & $66(74.2 \%)$ & $156(63.2 \%)$ & \\
\hline Titanium & $11(12.4 \%)$ & $31(12.6 \%)$ & \\
\hline End-stage renal disease & $1(1.1 \%)$ & $1(0.4 \%)$ & 0.435 \\
\hline Liver cirrhosis & $4(4.5 \%)$ & $2(0.8 \%)$ & 0.100 \\
\hline Impaired liver function & $11(12.4 \%)$ & $22(8.9 \%)$ & 0.519 \\
\hline Coagulopathy & $6(6.7 \%)$ & $8(3.2 \%)$ & 0.119 \\
\hline Diabetes mellitus & $14(15.7 \%)$ & $31(12.6 \%)$ & 0.546 \\
\hline Hypertension & $24(27.0 \%)$ & $69(27.9 \%)$ & 1.000 \\
\hline Intraop blood loss (ml) & $245.9 \pm 212.1$ & $196.4 \pm 167.6$ & 0.0497 \\
\hline Postop hemorrhage $\dagger$ & $5(5.6 \%)$ & $0(0.0)$ & 0.001 \\
\hline Postop infection & $5(5.6 \%)$ & $15(6.1 \%)$ & 0.375 \\
\hline No. of previous ops & $1.7 \pm 1.0$ & $1.8 \pm 1.4$ & 0.344 \\
\hline Repeat cranioplasty & $9(10.1 \%)$ & $23(9.3 \%)$ & 0.819 \\
\hline $\begin{array}{l}\text { Hydrocephalus before } \\
\text { cranioplasty }\end{array}$ & $32(36.0 \%)$ & $50(20.2 \%)$ & 0.014 \\
\hline $\begin{array}{l}\text { Hydrocephalus after } \\
\text { cranioplasty }\end{array}$ & $14(15.7 \%)$ & $17(6.9 \%)$ & 0.028 \\
\hline
\end{tabular}

$\mathrm{ICH}=$ intracerebral hemorrhage; $\mathrm{Sz}=$ seizure.

Values expressed as the mean \pm standard deviation or as the frequency (percent), unless indicated otherwise.

* Indicates $p<0.05$ in the Bonferroni-adjusted post hoc comparison.

$\dagger$ Hemorrhage includes epidural, subdural, and intraparenchymal hematoma requiring reoperation.

tive for seizure prophylaxis. No allergic or drug-related complications occurred in these 56 patients. After 1 week, AEDs were discontinued in all patients as no early seizures occurred. Sixteen (28.6\%) of the 56 patients had late seizures after cranioplasty. Overall, late-onset seizures occurred in 19\% (64/336) of the patients after cranioplasty. Eight (2.4\%) of the 336 patients suffered from new-onset seizures at 1 year postoperatively. The AEDs showed no protective effect against late seizures.

In the 247 patients who had preexisting seizures before cranioplasty, 106 (42.9\%) had frequent postoperative seizures and required an increased dosage of the AEDs
TABLE 2. Multivariable analysis for new-onset seizures

\begin{tabular}{lccc}
\hline \multicolumn{1}{c}{ Variable } & OR & $95 \% \mathrm{Cl}$ & $\mathrm{p}$ Value \\
\hline Liver cirrhosis & 7.27 & $1.14-46.58$ & 0.036 \\
\hline Intraop blood loss, per $10 \mathrm{ml}$ & 1.02 & $1.002-1.033$ & 0.024 \\
\hline Hydrocephalus before cranioplasty & 3.13 & $1.64-5.96$ & 0.001 \\
\hline Hydrocephalus after cranioplasty & 4.52 & $1.84-11.12$ & 0.001 \\
\hline
\end{tabular}

they were taking or the addition of new AEDs for seizure control. Only $57.1 \%$ patients who had preexisting seizures remained seizure free or had stable seizure control 2 years after cranioplasty.

\section{Discussion}

Cranioplasty is not merely a cosmetic operation, but provides protection to the intracranial contents from further injury and improves cerebral blood flow and neurological function. . $13,18,19,26$ However, postoperative seizures can lead to serious secondary complications, such as increased intracranial pressure and trauma from falling, resulting in further morbidity and even death. Cranioplasty after DC has been reported to be associated with a relatively high complication rate $(16.4 \%-34 \%)$ as compared to the rate after standard neurosurgical procedures $(2 \%-$ $5 \%) .{ }^{8}$ The reported incidence of postcranioplasty seizures varies from $8 \%$ to $33 \% .^{10,14,22,27}$ Our study showed a $7.4 \%$ $(25 / 336)$ early seizure rate and a new-onset seizure rate of $26.5 \%(89 / 336)$ in the long-term follow-up. In the patients with preexisting seizures before cranioplasty, the rate of postoperative seizures reached $42.9 \%$. Therefore, cranioplasty is associated with a relatively high incidence of postoperative seizures.

\section{Mechanisms of Postcranioplasty Seizures}

Seizures after cranioplasty may be the result of either the underlying brain condition or the cranioplasty procedure itself. In our study, $42.9 \%$ of patients with preexisting seizures before cranioplasty had postoperative seizures, which suggests that the underlying brain pathology affects the occurrence of seizures. The pathophysiology of early and late seizures is slightly different. Diffuse brain edema and ischemia lead to the release of excitatory cellular components and toxic mediators causing early seizures. ${ }^{12}$ Cranioplasty is considered an extradural procedure, and the manipulation of brain tissue during dissection of the extradural plane is unavoidable, which can result in brain edema. The dissection will also reduce focal cerebral blood flow because of the disconnection between the scalp and dura mater, which can cause relative ischemia in the underlying cortex. Moreover, brain tissue is further manipulated to facilitate the new contour of the bone flap after cranioplasty. These manipulations may precipitate seizure activity in already susceptible brain tissue and have been postulated to be mechanisms for postcranioplasty seizures. ${ }^{14,16}$ Late-onset seizures may be caused by a cascade of further events followed by hemorrhage, hemolysis, iron compound exportation, free radical formation, peroxidation, and cell death resulting in damage to the brain cortex and the formation of epileptogenic foci. ${ }^{6,21}$ 
TABLE 3. Prophylactic AED use in patients without preexisting seizures

\begin{tabular}{lccc}
\hline \multicolumn{1}{c}{ Variable } & AEDs $(n=56)$ & No AEDs $(n=280)$ & $p$ Value \\
\hline New-onset Szs $(n=89)$ & & 0.741 \\
\hline No & $40(71.4)$ & $207(73.9)$ & \\
\hline Yes & $16(28.6)$ & $73(26.1)$ & \\
\hline Early Sz $(n=25)$ & & & 0.012 \\
\hline No & $56(100.0)$ & $255(91.1)$ & \\
\hline Yes & $0(0.0)$ & $25(8.9)$ & \\
\hline Late Sz $(n=64)$ & & & 0.061 \\
\hline No & $40(71.4)$ & $232(82.9)$ & \\
\hline Yes & $16(28.6)$ & $48(17.1)$ & \\
\hline
\end{tabular}

Early $\mathrm{Sz}=$ seizure onset $\leq 7$ days after cranioplasty; late $\mathrm{Sz}=$ seizure onset $>$

7 days after cranioplasty; $n=$ total number of patients.

Boldface type indicates statistical significance.

Although the operation itself can also produce free radicals, disturb the ionic balance, and involve manipulation of the cerebral parenchyma, the cause of late-onset seizures is likely multifactorial..$^{14,27}$

\section{Prophylaxis Against Postcranioplasty Seizures}

A course of seizure prophylaxis for a week has only been well established for posttraumatic head injuries..$^{2,3,20}$ The incidence of postcranioplasty seizures is similar to, or even higher than, that seen with severe head injury. ${ }^{3,7}$ Therefore, the use of prophylactic AEDs should be considered for patients after cranioplasty. In this study, although the number of patients who received prophylactic AEDs for 1 week was only 56 , none of them had early seizures. The effect of early seizure prophylactics is obvious. There were no immediate or delayed adverse effects in the 56 patients, such as an allergic skin reaction, liver dysfunction, or impaired cognitive function. Phenytoin, sodium valproate, and levetiracetam are commonly used drugs, and all are effective at preventing post-TBI and postcraniotomy seizures., ${ }^{2,3,20} \mathrm{Al}-$ though the use of levetiracetam appears to be increasing for seizure prophylaxis, phenytoin (37/56 patients) is still the first choice at our center and is recommended to decrease the incidence of early posttraumatic seizures. ${ }^{3}$

\section{Risk Factors for Postcranioplasty Seizures}

Risk factors for postcranioplasty seizures have been reported to be TBI, hemorrhagic stroke, postoperative infection, postoperative hemorrhage, patient sex, and neurological deficits before operation. ${ }^{14,22,27}$ In our study, we found that intraoperative blood loss, postoperative hemorrhage, and hydrocephalus before or after cranioplasty were associated with seizures. After multivariable analysis, cirrhosis, intraoperative blood loss, and shunt-dependent hydrocephalus were predictors of postcranioplasty seizures. As shown in Table 1, the presence of a brain tumor reached statistical significance $(\mathrm{p}<0.05)$ in the univariable analysis but was not significant in the multivariable analysis. Brain tumors were present in 28 patients $(8.3 \%)$ in our study, but only 3 of these patients had new-onset seizures. It is likely that after tumor removal in the initial operation, the main seizure focus was removed. When these patients underwent cranioplasty, the rate of postoperative seizures was less than that associated with other etiologies. A prior randomized trial has suggested that brain tumor surgery itself does not have an influence on postoperative seizure and that prophylactic AEDs are unnecessary. ${ }^{24}$ In our study, only 10 patients had cirrhosis, and 4 of those with preexisting seizures before cranioplasty had more frequent seizures postoperatively. On the other hand, 4 of the 6 patients with cirrhosis and no seizures before cranioplasty had new-onset seizures. Thus, it seems advisable to carefully observe for new-onset or worsening seizures after cranioplasty in patients with cirrhosis. In such patients, seizures may result directly from the cranioplasty or from cirrhosis-related hepatic encephalopathy. ${ }^{4}$ Intraoperative blood loss and postoperative hemorrhage imply more brain manipulation and the new accumulation of hematoma after cranioplasty and re-switch on the process of hemolysis, iron exploration, and free radical releasing. These factors can lead to new-onset seizures after cranioplasty.

Our study showed that patients with hydrocephalus before or after cranioplasty have a higher risk of postoperative seizures. Hydrocephalus is well documented as a common complication after DC., ${ }^{9,16,25}$ When hydrocephalus develops in a patient after craniectomy, the brain may progressively bulge at the craniectomy site and a permanent shunt may be required. Cranioplasty has also been reported to contribute to the development of persistent hydrocephalus. ${ }^{17,23,27}$ Cranioplasty can affect the cerebral perfusion pressure and change the intracranial pressure. . $^{5,10}$ Performing cranioplasty in a sunken or bulging brain can create negative or positive pressure, respectively, and thus alter the intracranial pressure. That is why, clinically, adjusting the ventriculoperitoneal (VP) shunt pressure before cranioplasty, undergoing VP shunt insertion simultaneously with cranioplasty, or developing complications such as subdural fluid collection or subdural hematoma after cranioplasty occurs very frequently. Such brain pressure changes and complications could be caused or aggravated by CSF drainage and induce a seizure., ${ }^{922,23,27}$ We were unable to determine whether hydrocephalus was a direct complication of the primary brain injury, the craniectomy, or the cranioplasty. Thus, we suggest close surveillance for the development of hydrocephalus following cranioplasty.

\section{Limitations and Further Study}

The strength of this study is the long-term follow-up, the large sample size, and the single-center results without patient selection bias. However, the limitations of this study are the retrospective design and small number of patients who received prophylactic AEDs. In this study, only two attending neurosurgeons administered routine prophylactic AEDs for cranioplasty. Previous authors have suggested a new protocol for seizure prophylaxis based on their study results, ${ }^{22,27}$ however, seizure prophylaxis for cranioplasty is still not a common practice.

\section{Conclusions}

Cranioplasty is associated with a relatively high incidence of postoperative seizures. Patients who have underlying cirrhosis, more intraoperative blood loss, or shunt- 
dependent hydrocephalus are at greater risk for postcranioplasty seizures. Prophylactic use of AEDs for 1 week after cranioplasty can reduce the occurrence of early seizures. On average, the number needed to treat is 8.9 seizures (early seizure), which can be prevented with the administration of AEDs.

\section{Acknowledgments}

We thank Alfred Hsing-Fen Lin and Zoe Ya-Jhu Syu for assisting in the statistical analysis. We also acknowledge Gere Medical Writers Co. for editing this manuscript.

\section{References}

1. Agner C, Dujovny M, Gaviria M: Neurocognitive assessment before and after cranioplasty. Acta Neurochir (Wien) 144:1033-1040, 2002

2. Beenen LF, Lindeboom J, Kasteleijn-Nolst Trenité DG, Heimans JJ, Snoek FJ, Touw DJ, et al: Comparative double blind clinical trial of phenytoin and sodium valproate as anticonvulsant prophylaxis after craniotomy: efficacy, tolerability, and cognitive effects. J Neurol Neurosurg Psychiatry 67:474-480, 1999

3. Carney N, Totten AM, O'Reilly C, Ullman JS, Hawryluk GW, Bell MJ, et al: Guidelines for the management of severe traumatic brain injury, fourth edition. Neurosurgery 80:615,2017

4. Chen CC, Hsu PW, Lee ST, Chang CN, Wei KC, Wu CT, et al: Brain surgery in patients with liver cirrhosis. J Neurosurg 117:348-353, 2012

5. Dujovny M, Fernandez P, Alperin N, Betz W, Misra M, Mafee M: Post-cranioplasty cerebrospinal fluid hydrodynamic changes: magnetic resonance imaging quantitative analysis. Neurol Res 19:311-316, 1997

6. Englander J, Bushnik T, Duong TT, Cifu DX, Zafonte R, Wright J, et al: Analyzing risk factors for late posttraumatic seizures: a prospective, multicenter investigation. Arch Phys Med Rehabil 84:365-373, 2003

7. Ferguson PL, Smith GM, Wannamaker BB, Thurman DJ, Pickelsimer EE, Selassie AW: A population-based study of risk of epilepsy after hospitalization for traumatic brain injury. Epilepsia 51:891-898, 2010

8. Godil SS, Shamim MS, Enam SA, Qidwai U, Qadeer M, Sobani ZA: Cranial reconstruction after decompressive craniectomy: prediction of complications using fuzzy logic. J Craniofac Surg 22:1307-1311, 2011

9. Heo J, Park SQ, Cho SJ, Chang JC, Park HK: Evaluation of simultaneous cranioplasty and ventriculoperitoneal shunt procedures. J Neurosurg 121:313-318, 2014

10. Honeybul S, Janzen C, Kruger K, Ho KM: The impact of cranioplasty on neurological function. Br J Neurosurg 27:636-641, 2013

11. Honeybul S, Morrison DA, Ho KM, Lind CR, Geelhoed E: A randomized controlled trial comparing autologous cranioplasty with custom-made titanium cranioplasty. J Neurosurg 126:81-90, 2017

12. Khan AA, Banerjee A: The role of prophylactic anticonvulsants in moderate to severe head injury. Int J Emerg Med 3:187-191, 2010

13. Kuo JR, Wang CC, Chio CC, Cheng TJ: Neurological improvement after cranioplasty-analysis by transcranial Doppler ultrasonography. J Clin Neurosci 11:486-489, 2004

14. Lee L, Ker J, Quah BL, Chou N, Choy D, Yeo TT: A retrospective analysis and review of an institution's experience with the complications of cranioplasty. Br J Neurosurg 27:629-635, 2013

15. Liang S, Ding P, Zhang S, Zhang J, Zhang J, Wu Y: Prophy- lactic levetiracetam for seizure control after cranioplasty: a multicenter prospective controlled study. World Neurosurg 102:284-292, 2017

16. Liao CC, Kao MC: Cranioplasty for patients with severe depressed skull bone defect after cerebrospinal fluid shunting. J Clin Neurosci 9:553-555, 2002

17. Morton RP, Abecassis IJ, Hanson JF, Barber JK, Chen M, Kelly CM, et al: Timing of cranioplasty: a 10.75-year single-center analysis of 754 patients. J Neurosurg 128:1648-1652, 2018

18. Sakamoto S, Eguchi K, Kiura Y, Arita K, Kurisu K: CT perfusion imaging in the syndrome of the sinking skin flap before and after cranioplasty. Clin Neurol Neurosurg 108:583-585, 2006

19. Segal DH, Oppenheim JS, Murovic JA: Neurological recovery after cranioplasty. Neurosurgery 34:729-731, 1994

20. Temkin NR, Dikmen SS, Wilensky AJ, Keihm J, Chabal S, Winn HR: A randomized, double-blind study of phenytoin for the prevention of post-traumatic seizures. N Engl J Med 323:497-502, 1990

21. Thapa A, Chandra SP, Sinha S, Sreenivas V, Sharma BS, Tripathi M: Post-traumatic seizures-a prospective study from a tertiary level trauma center in a developing country. Seizure 19:211-216, 2010

22. Walcott BP, Kwon CS, Sheth SA, Fehnel CR, Koffie RM, Asaad WF, et al: Predictors of cranioplasty complications in stroke and trauma patients. J Neurosurg 118:757-762, 2013

23. Waziri A, Fusco D, Mayer SA, McKhann GM II, Connolly ES Jr: Postoperative hydrocephalus in patients undergoing decompressive hemicraniectomy for ischemic or hemorrhagic stroke. Neurosurgery 61:489-494, 2007

24. Wu AS, Trinh VT, Suki D, Graham S, Forman A, Weinberg JS, et al: A prospective randomized trial of perioperative seizure prophylaxis in patients with intraparenchymal brain tumors. J Neurosurg 118:873-883, 2013

25. Yang XF, Wen L, Shen F, Li G, Lou R, Liu WG, et al: Surgical complications secondary to decompressive craniectomy in patients with a head injury: a series of 108 consecutive cases. Acta Neurochir (Wien) 150:1241-1248, 2008

26. Yoshida K, Furuse M, Izawa A, Iizima N, Kuchiwaki H, Inao S: Dynamics of cerebral blood flow and metabolism in patients with cranioplasty as evaluated by $133 \mathrm{Xe}$ CT and $31 \mathrm{P}$ magnetic resonance spectroscopy. J Neurol Neurosurg Psychiatry 61:166-171, 1996

27. Zanaty M, Chalouhi N, Starke RM, Clark SW, Bovenzi CD, Saigh M, et al: Complications following cranioplasty: incidence and predictors in 348 cases. J Neurosurg 123:182188,2015

\section{Disclosures}

The authors report no conflict of interest concerning the materials or methods used in this study or the findings specified in this paper.

\section{Author Contributions}

Conception and design: Tu, Chen. Acquisition of data: Chen. Analysis and interpretation of data: Tu, Yeap, Chen, ZH Liu. Drafting the article: Yeap, Chen. Critically revising the article: $\mathrm{Tu}$, Yeap, Chen. Reviewed submitted version of manuscript: Tu, Chen, Liu, Hsieh. Approved the final version of the manuscript on behalf of all authors: Tu. Administrative/technical/material support: Chen, ZH Liu, Hsieh, Lee, YT Liu, Wang, Huang, Wei, Wu. Study supervision: Tu, ZH Liu, Wei, Wu.

\section{Correspondence}

Po-Hsun Tu: Chang Gung Memorial Hospital, Linkou Medical Center, Chang Gung Medical College \& University, Taoyuan City, Taiwan.d12096@cloud.cgmh.org.tw. 